\title{
PLURIPOTENCY
}

\section{Arrested development}

Cell Stem Cell 10, 171-182 (2012)

Although some extracellular ligands or intracellular regulators are known to affect pluripotency, the mechanisms that govern self-renewal in embryonic stem cells are not well understood. To investigate the relationship among known pathways, Li et al. evaluated the phosphorylation of ERK ( $p E R K$ ) in response to BMP4 or LIF, noting that sustained BMP4 treatment with or without LIF decreased pERK levels and that this response was specific to stem cells. To elucidate the connection between BMP4 signaling and pERK, the authors evaluated both the kinetics and the magnitude of the $p E R K$ response in the presence of the translational inhibitor cyclohexamide, demonstrating that new protein synthesis was required and occurred on a time frame consistent with new transcription. Previous chromatin immunoprecipitation sequencing studies had revealed transcriptional effectors downstream of BMP4 localized to the promoter of the Dusp9 gene, which encodes an ERK-specific phosphatase. In stem cells, Dusp9 expression increased in response to BMP4 but not other related ligands. Overexpression of Dusp 9 attenuated pERK, whereas expression of a dominantnegative Dusp9 or RNA interference-mediated knockdown of Dusp9 but not Dusp7 enhanced pERK. Dusp9 or small-molecule inhibitors of kinases upstream of ERK substituted for BMP4 to maintain ESC self-renewal. Taken together, these data suggest that, in combination with LIF, Dusp9 mediates BMP4 signaling to attenuate $\mathrm{pERK}$ and sustain pluripotency.

\section{RECEPTORS}

\section{GPCRs on a diet}

Neuron 73, 317-332 (2012)

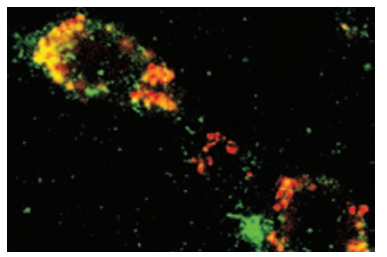

The G protein-coupled receptors growth hormone secretagogue receptor $1 \mathrm{a}$ (GHSR1a) and dopamine receptor subtype-2 (DRD2) are expressed in the mammalian brain and are linked to the anorexigenic response. However, the mode of action of GHSR1a is unknown, as its ligand ghrelin is barely detected in the brain. Now, Kern et al. provide evidence that unliganded GHSR1a allosterically modifies DRD2, affecting dopamine-mediated activation. Experiments in cell lines and primary neurons showed that expression of GHSR1a along with DRD2 was necessary for dopamine-induced $\mathrm{Ca}^{2+}$ mobilization. However, the downstream components of GHSR1a signaling were not necessary for DRD2 pathway activation, pointing to an interaction between the receptors rather than pathway crosstalk. Indeed, time-resolved fluorescence resonance energy transfer assays and confocal microscopy demonstrated that GHSR1a and DRD2 localized together into receptor heteromers at the cell surface and that alterations in the GHSR1a conformation, either by mutations or by treatment with antagonists, compromised DRD2-induced
$\mathrm{Ca}^{2+}$ mobilization. The authors verified the presence of endogenous GHSR1a-DRD2 heteromers in the hypothalamus of wildtype but not $\mathrm{Ghsr}^{-/-}$mice. In further support of a physiological role for the GHSR1aDRD2 heteromers, treatment of wild-type or ghrelin $^{-1-}$ mice with a DRD2 agonist resulted in anorexia, whereas the drug effect was blunted in $\mathrm{Ghsr}^{-/-}$mice. Conclusively, these results demonstrate that GPCR heteromer formation provides a new mechanism for controlling downstream signaling.

AC

\section{CARBOHYDRATES}

\section{cis gets with the program}

Angew. Chem. Int. Ed. Engl., published online 14 February 2012, doi:10.1002/anie.201108744

DNA and peptide synthesizers have made a tremendous impact on their fields by making available substantial amounts of precisely defined materials for further study. However, carbohydrate chemistry was long thought to be too complex to adapt to an automated protocol, given variability in monomer structures and desired connectivities. Efforts to automate 1,2-trans-glycosidic bond formation have made good progress, but it was unclear how the synthetically challenging 1,2-cis-glycosidic bond might be adapted to the solid phase. Walvoort et al. now provide the answer in their preparation of mannuronic acid alginates, molecules found in algal cell walls. The authors had previously observed that mannuronic acid donors formed cis linkages with high selectivity when used in short synthetic schemes, but these studies required monomer preactivation, a step that is not currently available in synthesizers and that uses chemistry not compatible with the resin linker. The authors instead picked $\mathrm{N}$-phenyltrifluoroacetimidate as a leaving group, which yielded the desired product exclusively when activated at $-40{ }^{\circ} \mathrm{C}$ with triflic acid. A temporary levulinoyl-ester protecting group could be removed under mild conditions, preparing the intermediate for the next step. After optimization of coupling efficiency, the authors generated tetrameric, octameric and dodecameric sequences, each with excellent stereoselectivity and with reaction yields of $90 \%$ at each step.

STRUCTURAL BIOLOGY

\section{Kinetochores ReWinD}

EMBO Rep., published online 10 February 2012, doi:10.1038/embor.2012.1

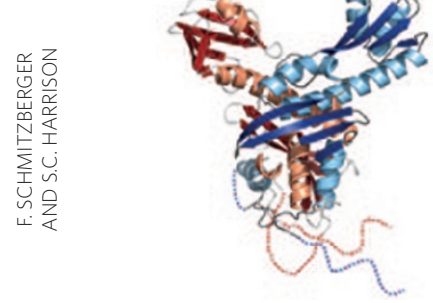

Eukaryotic kinetochores are molecular machines composed of multimeric complexes that attach the chromosomes to microtubules during cell division. A linker complex that bridges the complexes associated with the chromosomes and microtubules is essential. Schmitzberger and Harrison now report the structure of the linker protein subcomplex Ctf19-Mcm21, which belongs to the larger linker complex COMA. The authors observed that each protein forms two folded $\alpha+\beta$ sandwiches, which belong to the family of RWD domains, connected by an $\alpha$-helix of $\sim 40$ - $\AA$ length. The N-terminal $\alpha+\beta$ sandwiches form tight interprotein interactions, whereas the C-terminal ones protrude away from each other, forming a Y shape. The crystal structure and limited proteolysis showed that the $\mathrm{N}$-terminal residues of both proteins are unstructured and do not interact with each other or with other proteins of the linker complex. Truncation experiments indicated that the double RWD domains of the Ctf19-Mcm21 subcomplex are necessary and sufficient to interact with the larger COMA complex. Comparisons of the RWD domains of Ctf19-Mcm21 proteins with those of other kinetochore proteins revealed several other examples of these structural modules, pointing to the importance of the RWD domains in kinetochore architecture. 